Marquette University

e-Publications@Marquette

Mechanical Engineering Faculty Research and

Publications

Mechanical Engineering, Department of

9-14-2003

\title{
Admittance Selection for Planar Force-Guided Assembly for Single-Point Contact with Friction
}

Shuguang Huang

Marquette University, shuguang.huang@marquette.edu

Joseph M. Schimmels

Marquette University, joseph.schimmels@marquette.edu

Accepted version. Published as a part of the Proceedings. ICRA '03. IEEE International Conference on Robotics and Automation, 2003. DOI. (C) 2003 IEEE. Used with permission. 
Marquette University

e-Publications@Marquette

\title{
Mechanical Engineering Faculty Research and Publications/College of Engineering
}

This paper is NOT THE PUBLISHED VERSION; but the author's final, peer-reviewed manuscript. The published version may be accessed by following the link in the citation below.

IEEE Transactions on Robotics and Automation, Vol. 3, (2003): 3082-3088. DOI. This article is (C) Intitute of Electrical and Electronics Engineers (IEEE) and permission has been granted for this version to appear in e-Publications@Marquette. IEEE does not grant permission for this article to be further copied/distributed or hosted elsewhere without the express permission from IEEE.

\section{Admittance Selection for Planar Force-guided Assembly for Single-point Contact with Friction}

Shuguang Huang

Department of Mechanical Engineering, Marquette Univ., Milwaukee, WI

J.M. Schimmels

Department of Mechanical Engineering, Marquette Univ., Milwaukee, WI

\begin{abstract}
:
This paper identifies procedures for selecting the appropriate admittance to achieve reliable planar force-guided assembly for single-point frictional contact cases. A set of conditions that are imposed on the admittance matrix is presented. These conditions ensure that the motion that results from contact reduces part misalignment. We show that, for bounded misalignments, if an admittance satisfies the misalignment-reduction conditions at a finite number of contact configurations and a given coefficient of friction /spl mu//sub M/) then the admittance will also ensure that the conditions are satisfied at all intermediate configurations for all coefficients less than /spl mu//sub M/.
\end{abstract}




\section{SECTION I.}

\section{Introduction}

Admittance control has been used in assembly tasks to provide force regulation and force guidance. In robotic assembly tasks, the admittance maps contact forces into changes in the velocity of the body held by the manipulator. To achieve reliable assembly, the manipulator admittance must be appropriate for the particular assembly task. In this paper, we identify procedures used to select the appropriate manipulator admittance for planar assembly with friction.

We consider a simple form of admittance, a linear admittance control law. For planar applications, this admittance behavior has the form:

$$
\mathbf{v}=\mathbf{v}_{0}+\mathbf{A} \mathbf{w}_{(1)}
$$

where $\mathbf{v}_{0}$ is the nominal twist (a 3-vector for planar cases), $\mathbf{w}$ is the contact wrench (force and torque) measured in the body frame (a 3-vector), and $A$ is the admittance matrix (a $3 \times 3$ matrix).

Many researchers have addressed the use of admittance for force-guidance. Whitney ${ }^{[1], ~[2] ~ p r o p o s e d ~}$ that the compliance of a manipulator be structured so that contact forces lead to decreasing errors. Peshkin ${ }^{[3]}$ addressed the synthesis of an accommodation (inverse damping) matrix using least squared optimization. Asada ${ }^{[4]}$ used a similar unconstrained optimization. procedure for the design of an accommodation neural network.

A reliable admittance selection approach is to design the control law so that, at each possible part misalignment, the contact force always leads to a motion that reduces the existing misalignment. The approach is referred to as force-assembly and has been successful for workpart into fixture insertion when errors are infinitesimal ${ }^{[5], ~[6], ~[7] . ~}$

For force-assembly, the motion resulting from contact must instantaneously reduce misalignment. Since the configuration space of a rigid body is non-Euclidian, there is no "natural" metric for finite spatial error. In $^{[8]}$, several body-specific metrics are established. One metric is based on the Euclidean distance between a single point on the body and its location when properly positioned.

Previously, we have considered sufficient conditions on the admittance to ensure planar force-

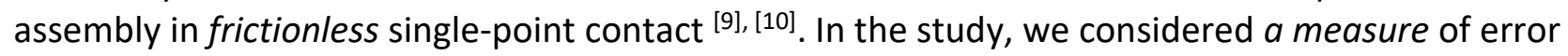
based on the Euclidean distance between a single (fixed) point on the held body and its location when properly positioned. The misalignment reduction condition of force-assembly requires that, at each possible misalignment, the contact force yields a motion that reduces the misalignment. Using the point-based measure of misalignment discussed above, this condition can be expressed mathematically if we let $d$ (a 3-vector for planar motion) be the line vector from the selected point at its proper mated position to its current position. Then, for error reducing motion, the condition is: 


$$
\mathbf{d}^{T} \mathbf{v}=\mathrm{d}^{T}\left(\mathbf{v}_{0}+\mathbf{A w}\right)<0
$$

which must be satisfied for all possible misalignments.

In this paper, we investigate single-point frictional contact using the same error measure. We show that, by identifying an admittance matrix that satisfies the error-reduction conditions at $a$ finite number of extremal contact configurations and at a specified coefficient of friction, the error reduction requirements are also satisfied for all intermediate configurations and for all coefficients of friction less than the one specified. The friction model considered is "hard" point contact satisfying Coulomb's law [11].

Planar bodies in single-point contact have two types of stable contact states. One is referred to as "vertexedge" contact $\{v-e\}$, Fig. 1a); the other is referred to as "edge-vertex" contact ( $\{e-v\}$, Fig. 1b).

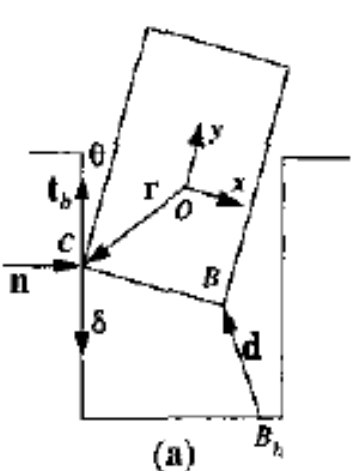

(a)

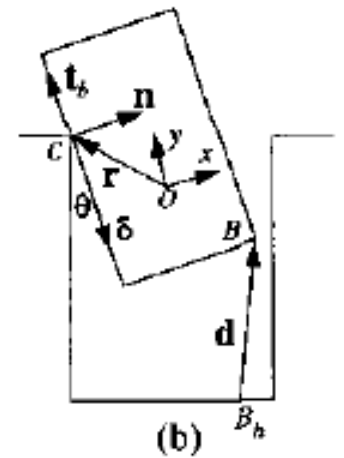

(b)

Fig. 1. Planar single-point contact. (a) Vertex-edge contact state. (b) Edge-vertex contact state.

In this paper, the motion of a rigid body constrained by a frictional contact is derived in Section II. Sufficient conditions for error reduction for vertex-edge and edge-vertex contact states are obtained in Section III and Section IV, respectively. These conditions show that an admittance matrix satisfying the error reduction conditions at the boundaries of a set of contact configurations and the coefficients of friction, also satisfies the error-reduction conditions at all intermediate configurations for all intermediate coefficients of friction. A brief summary is presented in Section V.

\section{SECTION II.}

\section{Motion of a Rigid Body Constrained by a Frictional Contact}

In this section, the planar motion a rigid body constrained by a single frictional surface is studied. First, the constrained compliant motion equation for frictional contact is derived. Then, the error-reduction function, describing the appropriate motion response in terms of the constraint and the admittance, is obtained. 


\section{A. Motion of a Constrained Rigid Body}

Consider a rigid body interacting with a frictional surface as shown in Fig. (1). Let $\boldsymbol{n}$ (unit 2-vector) be the surface normal (pointing toward the held body) and let $\mathbf{t}_{b}$ (unit 2-vector) be the unit vector tangent to the surface at the contact point. Then, the direction of friction $\mathrm{t}$ must be along $\mathbf{t}_{b}$, i.e., $\mathbf{t}=$ $\pm \mathbf{t}_{b}$.

The unit wrenches associated with the normal force and the friction force have the form:

$$
\mathbf{w}_{n}=\left[\begin{array}{c}
\mathbf{n} \\
(\mathbf{r} \times \mathbf{n}) \cdot \mathrm{k}
\end{array}\right], \mathbf{w}_{t}=\left[\begin{array}{c}
\mathbf{t} \\
(\mathbf{x} \times \mathbf{t}) \cdot \mathrm{k}
\end{array}\right]
$$

where $\boldsymbol{r}$ is the position vector from the origin of the coordinate frame to the point of contact, $c$, and $k$ is the unit vector orthogonal to the plane.

Let $\phi$ be the magnitude of the normal contact force. Since we only consider sliding motion, the overall contact wrench is:

$$
\mathbf{w}=\left(\mathbf{w}_{n}+\mu \mathbf{w}_{t}\right) \phi(4)
$$

where $\mu$ is the coefficient of friction.

By the control law (1), the motion of the body is:

$$
\mathbf{v}=\mathbf{v}_{0}+\mathbf{A}\left(\mathbf{w}_{n}+\mu \mathbf{w}_{t}\right) \phi \cdot(5)
$$

Due to "hard" point contact, the motion of the rigid body cannot penetrate the surface. Thus, the reciprocal condition ${ }^{[12]}$ must be satisfied:

$$
\mathbf{w}_{n}^{T} \mathbf{v}=\mathbf{w}_{n}^{T} \mathbf{v}_{0}+\mathbf{w}_{n}^{T} \mathbf{A}\left(\mathbf{w}_{n}+\mu \mathbf{w}_{t}\right) \phi=0 .
$$

The magnitude $\phi$ is determined from:

$$
\phi=\frac{-\mathbf{v}_{0}^{T} \mathbf{w}_{n}}{\mathbf{w}_{n}^{T} \mathbf{A} \mathbf{w}_{n}+\mu \mathbf{w}_{n}^{T} \mathbf{A} \mathbf{w}_{t}}
$$


Substituting (6) into $\underline{(5)}$ yields

$$
\mathbf{v}=\frac{\left(\mathbf{v}_{0} \mathbf{w}_{n}^{T}-\mathbf{v}_{0}^{T} \mathbf{w}_{n} \mathbf{I}\right) \mathbf{A}\left(\mathbf{w}_{n}+\mu \mathbf{w}_{t}\right)}{\mathbf{w}_{n}^{T} \mathbf{A} \mathbf{w}_{n}+\mu \mathbf{w}_{n}^{T} \mathbf{A} \mathbf{w}_{t}}
$$

where $I$ is the $3 \times 3$ identity matrix.

For planar motion, the normal $\boldsymbol{n}$ and tangent space base vector $\mathbf{t}_{b}$ at the contact point are known. The direction of the friction force $\left(t=t_{b}\right.$ ort $\left.=-t_{b}\right)$ is uniquely determined by satisfying the following conditions: 1) $\phi$ in (6) is positive, and 2) $\mathbf{v}^{T} \mathbf{t}<0$. Thus, $\boldsymbol{t}$ is known for a given contact point. The compliant motion can be determined by (7).

\section{B. Error-Reduction Function}

If the compliant motion is error-reducing, condition (2) must be satisfied for a given point. Thus,

$$
E=\frac{\mathbf{d}^{T}\left(\mathbf{v}_{0} \mathbf{w}_{n}^{T}-\mathbf{v}_{0}^{T} \mathbf{w}_{n} \mathbf{I}\right) \mathbf{A}\left(\mathbf{w}_{n}+\mu \mathbf{w}_{t}\right)}{\mathbf{w}_{n}^{T} \mathbf{A} \mathbf{w}_{n}+\mu \mathbf{w}_{n}^{T} \mathbf{A} \mathbf{w}_{t}}<0
$$

To avoid singularity in (7), the denominator must have no root over the range considered. Since $\mathbf{A}$ is positive definite, $\mathbf{w}_{n}^{T} \mathbf{A} \mathbf{w}_{n}>0$, the denominator is positive for $\mu=0$. Thus, we assume that, for $\mu \in$ $\left.\left[0, \mu_{M}\right]\right)$ the inequality

$$
\mathbf{w}_{n}^{T} \mathbf{A} \mathbf{w}_{n}+\mu \mathbf{w}_{n}^{T} \mathbf{A} \mathbf{w}_{t}>0
$$

is satisfied. Therefore, the error-reduction function can be expressed as:

$$
F_{e r}=\mathbf{d}^{T}\left(\mathbf{v}_{0} \mathbf{w}_{n}^{T}-\mathbf{v}_{0}^{T} \mathbf{w}_{n} \mathbf{I}\right) \mathbf{A}\left(\mathbf{w}_{n}+\mu \mathbf{w}_{t}\right) .
$$

In the following two sections, error-reduction conditions are obtained for the two single-point contact states. 


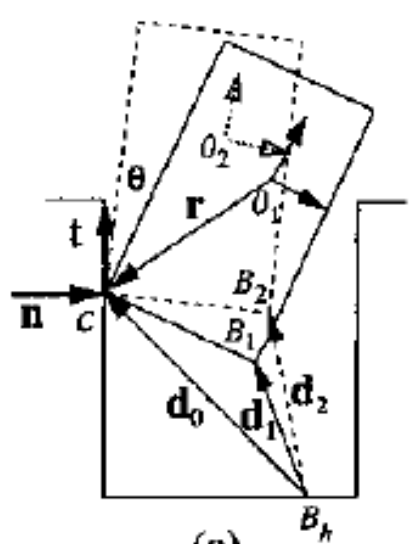

(a)

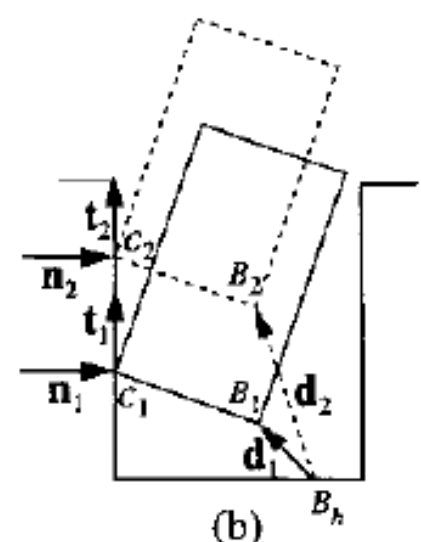

(b)

Fig. 2. Vertex-edge contact state. (a) Orientational variation. (b) Translational variation.

\section{SECTION III.}

\section{Vertex-edge Contact State}

In this section, vertex-edge contact is considered. As shown in Fig. 1a, the configuration of the body can be determined by the orientation of the body $\theta$ and the location of the contact point $\delta$.

Suppose that $\theta$ varies within the range of $\left|-\theta_{M}, \theta_{M}\right|$, and $\delta$ varies within the range of $\left[-\delta_{M}, \delta_{M}\right]$. We prove that, if an admittance matrix $A$ satisfies a set of conditions at a finite number of configurations for $\mu=0, \mu_{M}$, then the $A$ matrix ensures error-reducing motion for all configurations $\theta \in$ $\left[-\theta_{M}, \theta_{M}\right], \delta \in\left[-\delta_{M}, \delta_{M}\right]$, and all coefficients of friction $\mu \in\left[0, \mu_{M}\right]$.

Similar to the approach used for the frictionless case ${ }^{[9]}$, we first consider orientational and translational variation separately. Then, by combining the two variation cases, sufficient conditions for the general case are obtained.

\section{A. Orientational Variation}

Consider only orientation variation as illustrated in Fig. 2a. In this case, both the direction of the error reduction vector $\mathbf{d}$ and the direction of the contact wrench $w$ (in the body frame) are changed by changing the orientation. We prove that, for variation $\vartheta M \leq \pi 4$ if $A$ satisfies a set of conditions at orientation $\vartheta=0$, then an error-reducing motion is ensured for all configurations $\vartheta \in[-\vartheta M, \vartheta M]$.

\section{A1 Error Reduction Function}

Let $\mathbf{w}_{0}$ be the contact wrench, and $\mathrm{d}_{0}$ be the position vector associated with $\theta=0$. Suppose that at $\theta=0$, error-reducing motion is obtained, i.e., 


$$
\mathbf{d}_{0}^{T} \mathbf{v}_{0}+\mathbf{d}_{0}^{T} \mathbf{A} \mathbf{w}_{0}<0
$$

Consider a rotation given by an angle change $\theta$. Let $\mathbf{n}_{0}$ and $\mathbf{t}_{0}$ be the unit vectors in the directions of the normal force and friction force respectively when $\theta=0$, then in the body coordination frame, the two vectors associated with $\theta \in\left[-\theta_{M} \theta_{M}\right]$ are:

$$
\mathbf{n}_{\theta}=\mathbf{R}(\theta) \mathbf{n}_{0}, \mathrm{t}_{\theta}=\mathbf{R}(\theta) \mathrm{t}_{0}
$$

where $\boldsymbol{R}$ is the rotation matrix associated with $\theta$ having the form:

$$
\mathbf{R}(\theta)=\left[\begin{array}{ll}
\cos \theta & \sin \theta \\
\sin \theta & \cos \theta
\end{array}\right]
$$

The unit contact normal and friction wrenches calculated using $\underline{(3)}$ are:

$$
\begin{aligned}
& \mathbf{w}_{n}(\theta)=\left[\begin{array}{c}
\mathbf{R n}_{0} \\
\left(\mathbf{r} \times \mathbf{R n}_{0}\right) \cdot \mathrm{k}
\end{array}\right] \\
& \mathbf{w}_{t}(\theta)=\left[\begin{array}{c}
\mathbf{R t}_{0} \\
\left(\mathbf{r} \times \mathbf{R t}_{0}\right) \cdot \mathrm{k}
\end{array}\right]
\end{aligned}
$$

where $\boldsymbol{r}$ is the position vector from the origin of the body frame to the contact point (constant).

Since all configurations considered correspond to pure rotation about the contact point, the position vector of $B$ for an intermediate configuration can be expressed in the body frame as:

$$
\mathbf{d}_{\theta}^{\prime}=\mathbf{R} \mathbf{d}_{0}^{\prime}+\mathbf{d}^{\prime}(16)
$$

where $\mathbf{d}_{0}^{\prime}$ is the position vector from $B_{h}$ to the contact point $c$, $\mathbf{d}^{\prime}$ is the position vector from $c$ to point $B_{1}$. Note that $\mathbf{d}_{0}^{\prime}$ is a constant in the global frame and $\mathbf{d}^{\prime}$ is constant in the body frame. Then, the line vector of $B$ relative to its properly mated position $B_{h}$ (expressed in the body frame) is obtained:

$$
\mathbf{d}_{\theta}=\left[\begin{array}{c}
\mathbf{d}_{\theta}^{\prime} \\
\left(\mathbf{r}_{\theta} \times \mathbf{d}_{\theta}^{\prime}\right) \cdot \mathbf{k}
\end{array}\right]
$$


where $\mathrm{r}_{\theta}$ is the vector from the body frame origin to point $B$.

Since $\mathbf{d}_{\theta}, \mathbf{w}_{n}$ and $\mathbf{w}_{t}$ each involve first order terms in $\sin \theta$ and $\cos \theta$, the error-reduction function $(10)$ can be expressed as a third order polynomial in $\sin \theta$ and $\cos \theta$. Further, by the relation $\sin ^{2} \theta=1-$ $\cos ^{2} \theta$, the function can be written in the form:

$$
\begin{aligned}
F_{e r}(\theta)=\quad & c_{1} \cos ^{3} \theta+c_{2} \sin \theta \cos ^{2} \theta+c_{3} \cos ^{2} \theta+ \\
& c_{4} \sin \theta \cos \theta+c_{5} \sin \theta+c_{6} \cos \theta+c_{7}
\end{aligned}
$$

where the $c_{i}{ }^{\prime}$ s are functions of the admittance matrix $\mathbf{A}$ and the friction coefficient $\mu$ having the form:

$$
c_{i}=a_{i}+\mu b_{i}, i=1, \ldots, 7 \text {. (19) }
$$

\section{A2 Error Reduction Conditions}

To achieve error reduction at all other configurations and for any value of friction less than $\mu M$ considered, $F_{e r}(\theta)$ must be negative for all $\theta \in\left[-\theta_{M}, \theta_{M}\right]$ and $\mu \in\left[0, \mu_{M}\right]$. Now consider $F_{e r}$ as a function of $(\theta, \mu)$, then $F_{e r}(\theta, \mu)$ only contains a first order term in $\mu$.

In the following, we first obtain error reduction conditions for $\theta \in\left[-\theta_{M}, \theta_{M}\right]$ for both $\mu=0$ and $\mu=$ $\mu_{M}$. Then, we prove that the conditions for the extremal friction coefficients ensure error-reducing motion for any intermediate $\mu \in\left[0, \mu_{M} \mid\right.$.

By an appropriate rearrangement, $\underline{(18)}$ can be written as:

$$
\begin{gathered}
F_{e r}(\theta, \mu)=\left(c_{1} \cos ^{3} \theta+c_{3} \cos ^{2} \theta+c_{6} \cos \theta+c_{7}\right)+ \\
\left(c_{2} \cos ^{2} \theta+c_{4} \cos \theta+c_{5}\right) \sin \theta
\end{gathered}
$$

For $u=0, c_{i}=a_{i}$. A conservative "more positive" function $F_{0}^{+}(\theta)$ for $\theta>0$ is constructed based on (20) by the following

- If $a_{i}>0$, replace the corresponding $\cos \theta$ with 1 (by setting $\theta=0$ );

- If $a_{i}<0$, replace the corresponding $\cos \theta$ with $\cos \theta_{M}$.

As such, $F_{0}^{+}(\theta)$ has the form: 


$$
F_{0}^{+}(\theta)=a+a^{+} \sin \theta
$$

It can be seen that for any $0 \leq \theta \leq \theta_{M}$,

$$
\left.F(\theta)\right|_{\mu=0} \leq F_{0}^{+}(\theta)
$$

For $\theta<0$, a conservative "more positive" function $F_{0}^{-}(\theta)$, is constructed based on $\underline{(20)}$ by the following:

- For the terms involving $\sin \theta$, if $a_{i}>0$, replace the corresponding $\cos \theta$ with $\cos \theta_{M}$; if $a_{i}<$ 0 replace the corresponding $\cos \theta$ with 1 .

- For the terms involving only $\cos \theta$, if $a_{i}>0$, replace the corresponding $\cos \theta$ with 1 ; if $a_{i}<0$, replace the corresponding $\cos \theta$ with $\cos \theta_{M}$.

As such, $F_{0}^{-}$has the form:

$$
F_{0}^{-}(\theta)=a+a^{-} \sin \theta
$$

It can be seen that for any $-\theta_{M} \leq \theta \leq 0$,

$$
\left.F(\theta)\right|_{\mu=0} \leq F_{0}^{-}(\theta) \cdot(24)
$$

Because $\sin \theta$ is a monotonic function over $\left[-\frac{\pi}{4}, \frac{\pi}{4}\right], F_{0}^{+}(0)<0$, ensure that $F_{0}^{+}\left(\theta_{M}\right)<0$ for all $\theta \in$ $\left[0, \theta_{M}\right]$; and $F_{0}^{-}(0)<0$ and $F_{0}^{-}\left(-\theta_{M}\right)<0$ ensure that $F_{0}^{-}(\theta)<0$ for all $\theta \in\left[-\theta_{M}, 0\right]$. Since $F_{0}^{-}(0)=$ $F_{0}^{+}(0)$, the following set of 3 inequalities:

$$
\begin{gathered}
a<0 \\
a+a^{+} \sin \theta_{M}<0 \quad(25)(26)(27) \\
a-a^{-} \sin \theta_{M}<0
\end{gathered}
$$

ensures that $\left.F(\theta)\right|_{\mu=0}<0$ for all $\theta \in\left[-\theta_{M}, \theta_{M}\right]$. 
Using the same procedure for $\left.F(\theta)\right|_{\mu=\mu M}$, two conservative "more positive" function $F_{\mu_{M}}^{-}(\theta)$ and $F_{\mu_{M}}^{+}(\theta)$ are constructed:

$$
\begin{aligned}
& F_{\mu_{M}}^{+}(\theta)=e+e^{+} \sin \theta \\
& F_{\mu_{M}}^{-}(\theta)=e+e^{-} \sin \theta
\end{aligned}
$$

Thus, the following set of 3 inequalities:

$$
\begin{gathered}
e<0 \\
e+e^{+} \sin \theta_{M}<0 \quad(30)(31)(32) \\
e-e^{-} \sin \theta_{M}<0
\end{gathered}
$$

ensures that $\left.F(\theta)\right|_{\mu=\mu M}<0$ for all $\theta \in\left[-\theta_{M}, \theta_{M}\right]$.

Although inequalities (25)-(27) and (30)-(32) are constructed for two friction coefficients $\mu=0, \mu_{M}$, they are sufficient error reduction conditions for all $\mu \in[0, \mu M]$. In fact, since the error-reduction function $F$ contains only first order term of $\mu$, then, for any $\theta \in\left[-\theta_{M}, \theta_{M}\right]$ and $\mu \in\left[0, \mu_{M}\right]$,

$$
\begin{gathered}
\min \left\{F_{e r}(\theta, 0), F_{e r}\left(\theta, \mu_{M}\right)\right\} \leq F_{e r}(\theta, \mu) \\
\leq \max \left\{F_{e r}(\theta, 0), F_{e r}\left(\theta, \mu_{M}\right)\right\} .
\end{gathered}
$$

Since the sets of inequalities (25)-(27) and (30)-(32) ensure $F_{\text {er }}(\theta, 0)<0$ and $F_{\text {er }}\left(\theta, \mu_{M}\right)<0$, thus, from (33) $F_{\text {er }}(\theta, \mu)<0$ for $\forall \theta \in\left[-\theta_{M}, \theta_{M}\right], \forall \mu \in\left[0, \mu_{M}\right]$ is ensured by these inequalities.

\section{B. Translational Variation}

Now consider the translational variation of the contact configuration illustrated in Fig. $2 \mathrm{~b}$. In this case, only translation along the edge is allowed, and the contact force does not change in the body frame. The configuration of the body can be determined by a vector $\boldsymbol{d}$ (Fig. 2b).

Suppose that, at the two extremal configurations characterized by $\mathbf{d}_{1}$ and $\mathbf{d}_{2}$, the error reduction conditions are satisfied:

$$
\begin{aligned}
& \mathbf{d}_{1}^{T} \mathbf{v}_{0}+\mathbf{d}_{1}^{T} \mathbf{A} \mathbf{w}_{1}<0, \\
& \mathbf{d}_{2}^{T} \mathbf{v}_{0}+\mathbf{d}_{2}^{T} \mathbf{A} \mathbf{w}_{2}<0,
\end{aligned}
$$


where $\mathbf{w}_{1}$ and $\mathbf{w}_{2}$ are total contact wrenches at the two locations $c_{1}$ and $c_{2}$.

For any $\alpha, \beta \geq 0$,

$$
\left(\alpha \mathbf{d}_{1}+\beta \mathbf{d}_{2}\right)^{T} \mathbf{v}_{0}+\left(\alpha \mathbf{d}_{1}+\beta \mathbf{d}_{2}\right)^{T} \mathbf{A} \mathbf{w}<0
$$

At any intermediate configuration, the $\boldsymbol{d}$ vector is expressed as a convex combination of the vectors $\mathbf{d}_{1}$ and $\mathbf{d}_{2}$ i.e.,

$$
\mathbf{d}=\alpha \mathbf{d}_{1}+\beta \mathbf{d}_{2}
$$

where $\alpha, \beta>0$ and $\alpha+\beta=1$.

Since the contact wrench $\boldsymbol{w}$ is the same in the body frame for all contact configurations, $\mathbf{w}=\mathbf{w}_{1}=$ $\mathbf{w}_{2}$. Substituting $(37)$ into $(36)$ yields:

$$
F_{e r}=\mathbf{d}^{T} \mathbf{v}_{0}+\mathbf{d}^{T} \mathbf{A} \mathbf{w}<0
$$

Thus, for translational variation, if at two configurations the error reduction condition is satisfied, then the error reduction condition must be satisfied for all intermediate configurations bounded by these two configurations.

It is noted that the contact wrench $\mathbf{w}_{i}$ 's in $\underline{(34)}$ and $\underline{(35)}$ include friction. Because the coefficient of friction $\mu$ is linear in $F_{e r}$, satisfying the error-reduction conditions at $\mu=0, \mu_{M}$ ensures that the same conditions are satisfied for all $\mu \in\left[0, \mu_{M}\right]$.

\section{General Case}

Because of the linear dependence of the error-reduction function on the boundary configurations for the translational-only variation, similar to the frictionless case presented in ${ }^{[9]}$, the results presented in III-A and III-B can be generalized to the vertex-edge contact state involving both translational and orientational variations. Thus we have:

Proposition 1:

For a vertex-edge contact state with variation of orientation $\left[-\theta_{M}, \theta_{M}\right]$ and variation of translation $\left[-\delta_{M}, \delta_{M}\right]$, if at the two configurations with different contact boundary locations $\left[-\delta_{M}, \delta_{M}\right]$ the 
admittance satisfies inequalities (25)-(27) and (30)-(32) for $\mu=0$ and $\mu=\mu_{M}$, then the admittance will satisfy the error reduction condition for all configurations bounded by the four configurations, $\left[-\delta_{M},-\theta_{M}\right],\left[-\delta_{M}, \theta_{M}\right],\left[\delta_{M},-\theta_{M}\right],\left[\delta_{M}, \theta_{M}\right]$, for all $\mu \in\left[0, \mu_{M}\right]$.

Therefore, for an edge-vertex contact state, to ensure that the motion response due to contact is error reducing for all configurations considered, function values at only two configuration extremals and two coefficients of friction need be tested.

\section{SECTION IV.}

\section{Edge-vertex Contact State}

Consider "edge-vertex" contact. As shown in Fig. 1b, the configuration of the body can be determined by two parameters, $(\delta, \theta)$.

Suppose that $\theta$ varies within the range of $\left[-\theta_{M}, \theta_{M}\right]$, and $\delta$ varies within the range of $\left[-\delta_{M}, \delta_{M}\right]$. We prove that, if an admittance matrix $\mathbf{A}$ satisfies a set of conditions at the "boundary" points for $\mu=0$ and $\mu_{M}$, then the $\mathbf{A}$ matrix ensures error $-\theta \in\left[-\theta_{M}, \theta_{M}\right], \delta \in\left[-\delta_{M}, \delta_{M}\right]$, and $\mu \in\left[0, \mu_{M}\right]$.

In this case, the error-reduction function does not linearly depend on the configuration parameter $\theta$ or $\delta$ when considering either orientational or translational variation separately. As a consequence, a somewhat more complicated evaluation is used in which the orientational and translational variation are considered simultaneously.

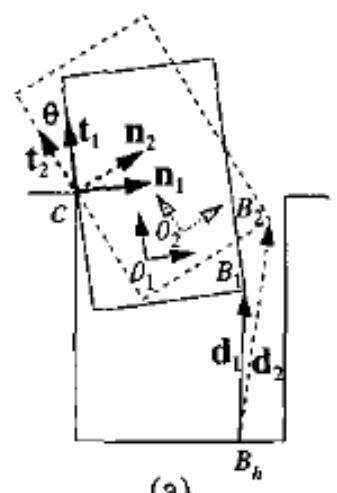

(a)

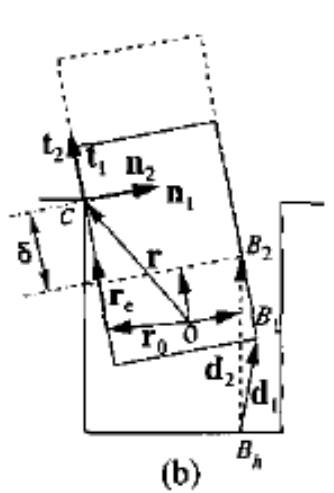

(b)

Fig. 3. Edge-vertex contact state. (a) Orientational variation: the contact wrench $w$ is constant in the body frame while the error-measure vector $\boldsymbol{d}$ is a nonlinear function of $\theta$. (b) Translational variation: both the contact wrench $w$ and the error-measure vector $\boldsymbol{d}$ are functions of $\delta$.

\section{A. Error-Reduction Function}

In order to obtain the error-reduction function, we first express the contact wrench and the errormeasure vector $\boldsymbol{d}$ in terms of $\delta$ and $\theta$. 
For an edge-vertex contact state, as shown in Fig. 3a, when the held body rotates relative to the fixtured body about the contact point, the description of the contact wrench does not change in a body-based coordinate frame. When the held body translates relative to the fixtured body, the description of the contact wrench changes in a body-based coordinate frame as the contact point changes (although its direction is constant). Thus, the contact force is a function involving only the translational variable $\delta$.

As shown in Fig. 3b, in the body frame, the direction of the surface normal is constant while the position vector of the contact point, $\boldsymbol{r}$, varies. For an arbitrary $\delta, \mathbf{r}$ can be expressed as:

$$
\mathbf{r}_{\delta}=\mathbf{r}_{0}+\mathbf{r}_{e} \delta
$$

where $\mathbf{r}_{0}$ is a vector from the body frame to a center point of the edge (constant) and $\mathbf{r}_{e}$ is the unit vector along the edge.

By (3), the unit wrench corresponding to the surface normal and friction are:

$$
\mathbf{w}_{n}=\left[\begin{array}{c}
\mathbf{n} \\
\left(\mathbf{r}_{\delta} \times \mathbf{n}\right) \cdot \mathrm{k}
\end{array}\right], \mathbf{w}_{t}=\left[\begin{array}{c}
\mathbf{t} \\
\left(\mathbf{r}_{\delta} \times \mathbf{t}\right) \cdot \mathrm{k}
\end{array}\right]
$$

It can be seen that in the body frame, the directions of $\mathbf{w}_{n}$ and $\mathbf{w}_{t}$ are constant while the last components (the moment terms) are linear functions of $\delta$.

Let $\mathbf{d}_{0}^{\prime}$ be the error-measure 2-vector at $(\theta, \delta)=(0,0)$, then for an arbitrary $\delta$ with $\theta=0$, the errormeasure vector $\mathrm{d}^{\prime}$ is:

$$
\mathbf{d}_{\delta}^{\prime}=\mathbf{d}_{0}^{\prime}+\mathbf{r}_{e} \delta, \delta \in\left[-\delta_{M}, \delta_{M}\right]
$$

where $\mathbf{r}_{\mathrm{e}}$ is a unit vector along the contacting edge. Note that $\boldsymbol{d}^{\prime} 0$ is constant in the global coordinate frame while $\mathbf{r}_{\mathrm{e}}$ is constant in body coordinate frame. Thus for an arbitrary orientation $\theta \in\left[-\theta_{M}, \theta_{M}\right]$ and $\delta \in\left[-\delta_{M}, \delta_{M}\right]$, the error-measure 2-vector $\mathbf{d}^{\prime}$ is a function of $\delta$ and $\theta$ having the form:

$$
\mathbf{d}^{\prime}(\delta, \theta)=\mathbf{R d}_{0}^{\prime}+\mathbf{r}_{e} \delta,(41)
$$

where $\boldsymbol{R}$ is the rotation matrix having the form of $\underline{(13)}$.

The line vector associated with $\mathbf{d}^{\prime}(\delta, \theta)$ can be calculated: 


$$
\mathbf{d}(\delta, \theta)=\left[\begin{array}{c}
\mathbf{R} \mathbf{d}_{0}^{\prime} \\
\left(\mathrm{r}_{B} \times \mathbf{R d}_{0}^{\prime}\right) \cdot \mathrm{k}
\end{array}\right]+\delta\left[\begin{array}{c}
\mathbf{r}_{e} \\
\left(\mathbf{r}_{B} \times \mathbf{r}_{e}\right) \cdot \mathrm{k}
\end{array}\right]
$$

where $\mathbf{r}_{B}$ is the position vector from the body frame origin to point $B$.

Thus, for any intermediate configuration $(\delta, \theta)$, b e-cause $\mathbf{w}_{n}$ and $\mathbf{w}_{t}$ in (39) each only contain first order terms in $\delta$ and $\mathrm{d}(\delta, \theta)$ in (42) only contains first order terms in $\sin \theta, \cos \theta$ and $\delta$, the errorreduction function (10) can be expressed as a third order polynomial in $\delta$ in the form:

$$
F_{e r}(\delta, \theta)=f_{3} \delta^{3}+f_{2} \delta^{2}+f_{1} \delta+f_{0}
$$

where the coefficients $f_{i}$ 's have the form:

$$
f_{i}=a_{i} \cos \theta+b_{i} \sin \theta
$$

Also note that, $\mu$ appears in the coefficients of $\mathbf{w}_{t}$. Therefore, the coefficients $a_{i}$ and $b_{i}$ have the form:

$$
a_{i}=\left(p_{i}+\mu p_{i}^{\prime}\right), b_{i}=\left(q_{i}+\mu q_{i}^{\prime}\right)
$$

where $p_{i}, p_{i}^{\prime}, q_{i}$ and $q_{i}^{\prime}$ are functions of the admittance $\mathrm{A}$.

\section{B. Sufficient Conditions for Error-Reduction}

The error-reduction condition requires that the error-reduction function in (43) must be negative in the range of configurations considered. In order to obtain sufficient conditions, we construct two functions $F_{0}$ and $F_{M}$ by replacing the $\cos \theta$ terms in (45) with 1 and $\cos \theta_{M}$ respectively:

$$
\begin{aligned}
& F_{0}(\delta, \theta)=\left(a_{3} \delta^{3}+a_{2} \delta^{2}+a_{1} \delta+a_{0}\right)+ \\
&\left(b_{3} \delta^{3}+b_{2} \delta^{2}+b_{1} \delta+b_{0}\right) \sin \theta \\
& F_{M}(\delta, \theta)=\left(a_{3} \delta^{3}+a_{2} \delta^{2}+a_{1} \delta+a_{0}\right) \cos \theta_{M}+ \\
&\left(b_{3} \delta^{3}+b_{2} \delta^{2}+b_{1} \delta+b_{0}\right) \sin \theta
\end{aligned}
$$

For small $\theta$ (e.g. , $\left.\theta \leq \frac{\pi}{8}\right), F_{0}$ and $F_{M}$ are close approximations of $F_{e r}$, and for any $(\delta, \theta)$ in the range considered, 


$$
\min \left\{F_{0}, F_{M}\right\} \leq F_{e r} \leq \max \left\{F_{0}, F_{M}\right\}
$$

Thus, if both $F_{0}$ and $F_{M}$ are negative over the range $\delta \in\left[-\delta_{M}, \delta_{M}\right]$ and $\theta \in\left[-\theta_{M}, \theta_{M}\right]$, error reducing motion is ensured.

For a given $\theta$, both $F_{0}$ and $F_{M}$ are third order polynomials in $\delta$, To obtain conditions on $F_{0}$ and $F_{M}$, we first evaluate the bounds on the coefficients of these two polynomials.

By $(46)$ and (47), the coefficients of $\delta^{i}$ in $F_{0}$ and $F_{M}$ are:

$$
\begin{aligned}
& f_{i}^{0}(\mu, \theta)=\left(p_{i}+p_{i}^{\prime} \mu\right)+\left(q_{i}+q_{i}^{\prime} \mu\right) \sin \theta \\
& f_{i}^{M}(\mu, \theta)=\left(p_{i}+p_{i}^{\prime} \mu\right) \cos \theta_{M}+\left(q_{i}+q_{i}^{\prime} \mu\right) \sin \theta .
\end{aligned}
$$

If the range of $\mu$ is $\left[0, \mu_{M}\right]$, it can be proved that $f_{i}^{0}$ and $f_{i}^{M}$ achieve their maximum and minimum values only at the boundary points $\left(0, \pm \theta_{M}\right)$ and $\left(\mu_{M}, \pm \theta_{M}\right)$. This can be verified by evaluating the Hessian matrices of $f_{i}^{0}$ and $f_{i}{ }^{M}$. In fact, the Hessian matrix of $f 0 i$ with respect to $(\mu, \theta)$ is:

$$
\operatorname{Hess}\left(f_{i}^{0}\right)=\left[\begin{array}{cc}
0 & q_{i}^{\prime} \cos \theta \\
q_{i}^{\prime} \cos \theta & -\left(q_{i}+q_{i}^{\prime} \mu\right) \sin \theta
\end{array}\right] .
$$

Since for $|\theta| \leq \frac{\pi}{8}, \operatorname{det}($ Hess $)=-q_{i}^{2} \cos \theta<0$, the Hessian is indefinite and the function $f_{i}^{0}$ cannot have a maximum or minimum in the interior of the area $\left[0, \mu_{M}\right] \times\left[-\theta_{M}, \theta_{M}\right]^{[13]}$. Thus, the maximum (minimum) values of $f_{i}^{0}$ can be chosen from its four values at the 4 boundary points: $\left(0, \pm \theta_{M}\right)$ and $\left(\mu_{M}, \pm \theta_{M}\right)$. The same property holds true for $f_{i}^{M}$.

Denote

$$
\begin{aligned}
s_{M} & =\max \left\{\left|f_{i}^{0}\right|,\left|f_{i}^{M}\right|, i=1,2,3\right\}, \\
s_{0} & =\min \left\{\left|f_{0}^{0}\right|,\left|f_{0}^{M}\right|\right\} .
\end{aligned}
$$

We prove that if

$$
\frac{s_{0}}{s_{M}+s_{0}}>\delta_{M}
$$


then both $F_{0}$ and $F_{M}$ have no root for all $\delta \in\left[-\delta_{M}, \delta_{M}\right], \theta \in\left[-\theta_{M}, \theta_{M}\right]$. and $u \in\left[0, \mu_{M}\right]$.

Consider the function $F_{0}$ in (46). For an arbitrary $\theta_{0} \in\left[-\theta_{M}, \theta_{M}\right]$ and an arbitrary $\mu_{0} \in\left[0, \mu_{M}\right], F_{0}$ is a third order polynomial in a single-variable $\delta$ :

$$
F_{0}\left(\delta, \theta_{0}\right)=c_{3} \delta^{3}+c_{2} \delta^{2}+c_{1} \delta+c_{0}
$$

Where

$$
c_{i}=\left(p_{i}+\mu_{0} p_{i}^{\prime}\right)+\left(q_{i}+\mu_{0} q_{i}^{\prime}\right) \sin \theta_{0} \cdot \text { (55) }
$$

Let

$$
c_{M}=\max \left\{\left|c_{1}\right|,\left|c_{2}\right|,\left|c_{3}\right|\right\},(56)
$$

then, as shown in ${ }^{[10]}$, a root of $F_{0}, \xi$, must satisfy

$$
|\xi| \geq \frac{\left|c_{0}\right|}{c_{M}+\left|c_{0}\right|}
$$

Since $\theta_{0} \in\left[-\theta_{M}, \theta_{M}\right]$ and $\mu 0 \in\left[0, \mu_{M}\right]$, by (51) and (52), we have:

$$
c_{M} \leq s_{M},\left|c_{0}\right| \geq s_{0} \cdot(58)
$$

Therefore,

$$
\frac{s_{M}}{s_{0}} \geq \frac{c_{M}}{c_{0}}(59)
$$

which leads to

$$
|\xi| \geq \frac{\left|c_{0}\right|}{c_{M}+\left|c_{0}\right|} \geq \frac{s_{0}}{s_{M}+s_{0}}>\delta_{M \cdot(60)}
$$


Thus, $F_{0}$ has no root in $\left[-\delta_{M}, \delta_{M}\right]$ for all $\theta \in\left[-\theta_{M}, \theta_{M}\right]$ and $\mu \in\left[0, \mu_{M}\right]$. The same reasoning applies to $F_{M}$. Therefore, the functions $F_{0}$ and $F_{M}$ do not change sign if inequality (53) is satisfied. By (48), Fer has no root in the same bounded area. Since the $s_{M}$ in (51) and $s_{0}$ in (52) are functions of the admittance A, (53) imposes a constraint on A. In summary, we have:

\section{Proposition 2:}

For an edge-vertex contact state, if: i) at the configuration $[\delta, \theta]=[0,0]$, the admittance satisfies the error reduction condition (2), and ii) condition (53) is satisfied for the configuration boundary points $[ \pm \delta, \pm \theta]$ and the maximum value of friction coefficient $\mu_{M}$, then the admittance will satisfy the error reduction conditions for all configurations bounded by these four configurations and friction coefficient $\mu \leq \mu_{M}$.

Thus, for an edge-vertex contact state, to ensure that contact yields error-reducing motion for the body, only four configuration extremals at two extremal coefficients of friction need be tested.

\section{SECTION V.}

\section{Summary}

In this paper, the error reduction condition for a single point on the held body in frictional contact is considered. We have presented an approach for admittance selection of a planar rigid body motion for force-guided assembly with friction. We have shown that, for one point contact cases, the admittance control law can be selected based on their behavior at a finite number of configurations and at two extremal coefficients of friction of the contact. If the error reduction conditions are satisfied at these configurations with these two coefficients of friction, error reduction will be satisfied for all intermediate configurations and all intermediate coefficients of friction. Thus, for a given set of bounded misalignments, a single admittance control law that satisfies these conditions guarantees the proper assembly of a given pair of mating parts.

\section{ACKNOWLEDGMENTS}

This research was supported in part by a Ford Motor Company URP award and the National Science Foundation under grant IIS 0010017.

\section{References}

${ }^{1}$ D. E. Whitney, "Force feedback control of manipulator fine motions," ASME Journal of Dynamic Systems, Measurements and Control, vol. 98, no. 2, 1977.

2 D. E. Whitney, "Quasi-static assembly of compliantly supported rigid parts," ASME Journal of Dynamic Systems, Measurements, and Control, vol. 104, no. 1, pp. 65-77, 1982. 
${ }^{3}$ M. A. Peshkin, "Programmed compliance for error-corrective manipulation," IEEE Transactions on Robotics and Automation, vol. 6, no. 4, pp. 473-482, 1990.

${ }^{4} \mathrm{H}$. Asada, "Teaching and learning of compliance using neural net," IEEE Transactions on Robotics and Automation, vol. 9, no. 6, pp. 863-867, 1993.

5 J. M. Schimmels and M.A. Peshkin, "Admittance matrix design for force guided assembly," IEEE Transactions on Robotics and Automation, vol. 8, no. 2, pp. 213-227, 1992.

${ }^{6}$ J. M. Schimmels and M. A. Peshkin, "Force-assembly with friction," IEEE Transactions on Robotics and Automation, vol. 10, no. 4, pp. 465-479, 1994.

7 J. M. Schimmels, "A linear space of admittance control laws that guarantees force-assembly with friction," IEEE Transactions on Robotics and Automation, vol. 13, no. 5, pp. 656-667, 1997.

8 J. M. R. Martinez and J. Duffy, "On the metric of rigid body displacements for infinite and finite bodies," ASME Journal of Mechanical Design, vol. 117, no. 1, pp. 41-47, 1995.

${ }^{9}$ S. Huang and J. M. Schimmels, "Sufficient conditions used in admittance selection for planar forceguided assembly," in Proceedings of the IEEE International Conference on Robotics and Automation, Washington, D.C., May 2002, pp. 538-543.

${ }^{10} \mathrm{~S}$. Huang and J. M. Schimmels, "Sufficient conditions used in admittance selection for force-guided assembly of polygonal parts," IEEE Transactions on Robotics and Automation, (submitted for publication).

${ }^{11} \mathrm{~S}$. Huang and J. M. Schimmels, "The motion of a compliantly suspended rigid body constrained by a frictional surface," in Proceedings of the ASME International Congress and Exposition, New York, NY, November 2001, vol. 2.

12 M. S. Ohwovoriole and B. Roth, "An extension of screw theory," ASME Journal of Mechanical Design, vol. 103, no. 4, pp. 725-735, 1981.

13 G. A. Korn, Mathematical Handbook for Scientists and Engineers, McGraw-Hill Book, Inc., 1968. 